\title{
Energy spectra of secondary electrons induced by fast ions under channeling conditions
}

\author{
H. Kudo and K. Shima \\ Institute of Applied Physics, University of Tsukuba, Ibaraki 305, Japan \\ S. Seki \\ Tandem Accelerator Center, University of Tsukuba, Ibaraki 305, Japan \\ K. Takita, K. Masuda, K. Murakami, and T. Ipposhi \\ Institute of Materials Science, University of Tsukuba, Ibaraki 305, Japan \\ (Received 10 August 1987; revised manuscript received 21 December 1987)
}

\begin{abstract}
We have studied simple aspects of the energy spectra of ion-induced secondary electrons emitted from single crystals under channeling conditions. The energy spectra for Si and GaAs targets, measured at a backward angle of $180^{\circ}$ for various incident ions over a 2-8 MeV/amu energy range, showed a constant decrease in the electron yield over certain $\mathrm{keV}$ energy regions when channeling occurred. This behavior was interpreted as a decrease in the effective target thickness, resulting from the ion-beam shadowing effect near crystal surfaces.
\end{abstract}

\section{INTRODUCTION}

Since the first observation by Magnuson and Carlston, ${ }^{1}$ several authors ${ }^{2-4}$ have reported that the ion-induced emission of secondary electrons from single-crystal targets strongly depends on the incident direction of projectile ions. By using low-energy (less than $100 \mathrm{keV}$ ) ions, they found that the total electron yield reflects the structure of the atomic rows or planes that are parallel to the ion-beam direction. This effect, however, has not been precisely studied mainly because of the difficulty of treating the complicated scattering of electrons in solids, which produces the observed secondary electrons. This situation is in contrast to simplicity in the case of Rutherford backscattering or characteristic x-ray emission under channeling conditions. ${ }^{5}$

This phenomenon should be elucidated by analyzing the energy spectra of the emitted electrons. Such energy spectra under channeling (shadowing) conditions have been measured recently by MacDonald et al., ${ }^{6}$ and by Kudo et al. $^{7-9}$ with particular interest in the emission of Auger electrons. Typically, the observed electron yields for channeling incidences showed a marked decrease in the high-energy region (roughly 50\% decrease for lowindex axial channeling). However, this decrease has not been accounted for in those papers. It is still unknown how the decrease in the electron yield under channeling conditions depends, e.g., on the structure of the crystal channel, and on the ion species.

This paper reports on an experimental study of the electron emission under channeling conditions not only with fundamental, but with technical interest in the structure analysis of thin surface layers by using secondary electrons as a probe.

\section{EXPERIMENTAL}

Figure 1 schematically shows the apparatus used in this experiment, which is similar to that described previ- ously $^{8}$ except for the newly designed electron spectrometer. As illustrated in Fig. 1, the $45^{\circ}$ parallel-plate electrostatic spectrometer of the mirror-symmetry type is capable of providing a variable energy acceptance by adjusting the window slit from outside the target chamber. This mechanism is useful for channeling experiments since the choice of a wide energy acceptance enables quick alignment of the crystal direction under high count rates (typically 2000 counts/sec). The energy spectra of electrons were measured with a relative energy resolution of typically $5 \%$ by setting the window width at $2-3 \mathrm{~mm}$. The spectra measured with the previous low-background spectrometer were well reproduced using the new spectrometer, which ensures the effective suppression of stray electrons in the new spectrometer. Since the electrons were analyzed in energy at $180^{\circ}$ (over a solid angle of about $2 \times 10^{-3} \mathrm{sr}$ ) with respect to the ion-beam direction,

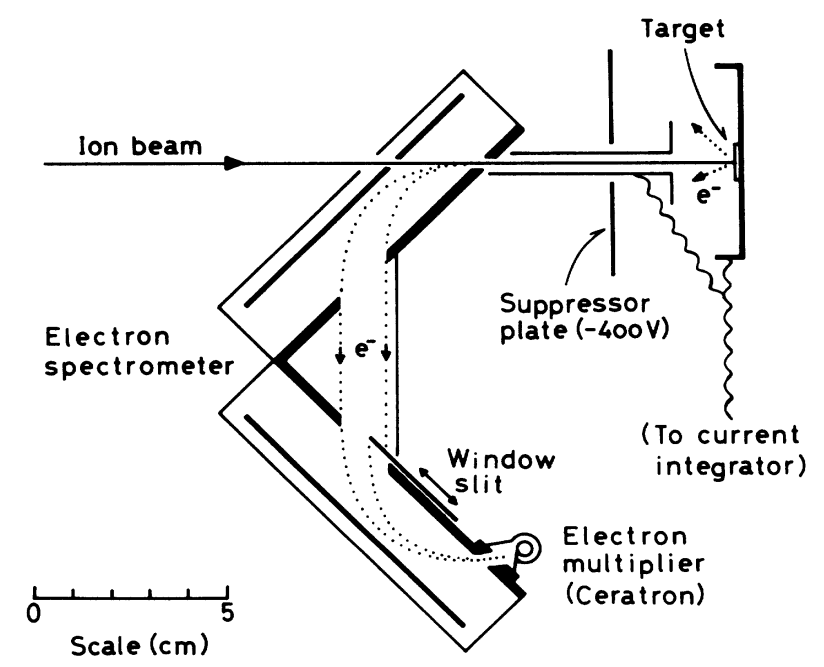

FIG. 1. Schematic diagram of the experimental arrangement. In the spectrometer, the analyzed electrons are focused on the same entrance position into the electron multiplier. 
no error associated with misalignment of the spectrometer axis was detected. ${ }^{8}$ Actually, tilting of the sample surface up to $20^{\circ}$ caused no noticeable change in the electron yield measured for nonchanneling incidences of projectile ions.

The (110), (111), and (100) wafers of Si crystals (0.3-0.5 $\mathrm{mm}$ thick) and (100) wafers of GaAs crystals $(0.3 \mathrm{~mm}$ thick) were chemically etched. Under our experimental conditions, the surfaces are covered with contaminants of a few monolayers of carbon and oxygen atoms, as identified previously. ${ }^{8}$ Such thin layers, however, hardly affect the decrease in the high-energy electron yield since under channeling conditions those electrons originate from collisions between the projectile and target electrons in the surface region thicker than about $150 \AA$, as will be estimated later (Sec. IV D and Fig. 9).

The measurements were carried out at room temperature (290-295 K) under the vacuum conditions of about $5 \times 10^{-6} \mathrm{~Pa}$ in pressure. The ion beams were obtained from the Pelletron Tandem Accelerator at the University of Tsukuba. The beam current was 10-20 nA irrespective of the ion species used. The electron-energy spectra showed no noticeable influence of beam irradiation under the present beam dose ranging from $10^{14}$ to $10^{16}$ ions $/ \mathrm{cm}^{2}$.

\section{RESULTS}

The energy spectra presented are raw data, i.e., the electron yield is the number of electrons counted and it includes no correction for the energy dependence of the spectrometer's acceptance. ${ }^{10}$

Figure 2 shows energy spectra of secondary electrons induced by $30-\mathrm{MeV} \mathrm{O}^{5+}$, measured for $\langle 110\rangle$ channeling and random (nonchanneling) incidences on Si. A remarkable decrease in the electron yields is seen for the channeling case. Figure 3 shows the normalized electron

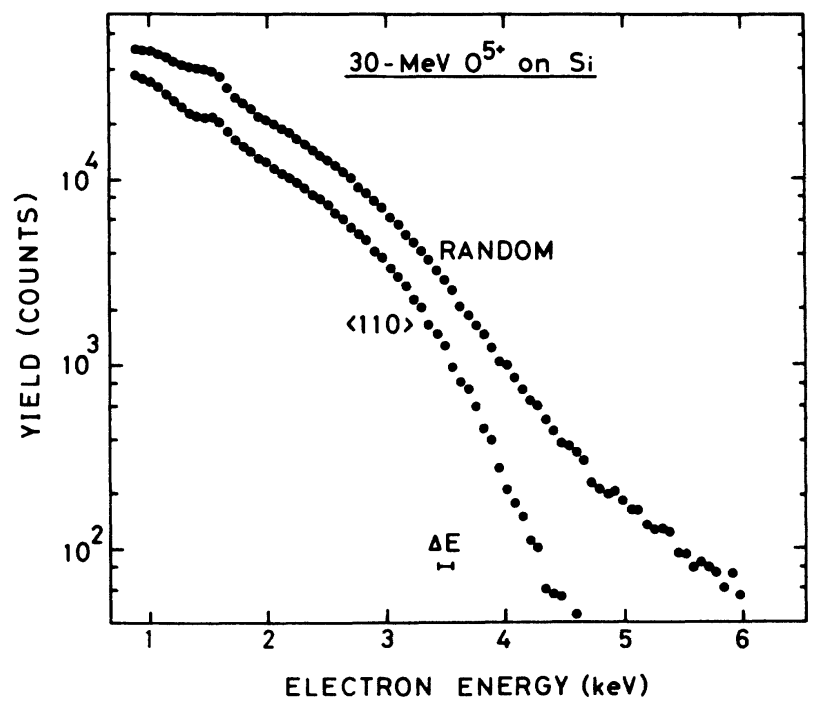

FIG. 2. Energy spectra of secondary electrons induced by $30-\mathrm{MeV} \mathrm{O}^{5+}$ under $\mathrm{Si}\langle 110\rangle$ channeling and random conditions, measured at a backward angle of $180^{\circ}$ for same number (per channel) of incident ions. The peaks near $1.6 \mathrm{keV}$ are due to Si $K$-shell Auger electrons. $\Delta E$ represents the spectrometer's relative energy resolution.

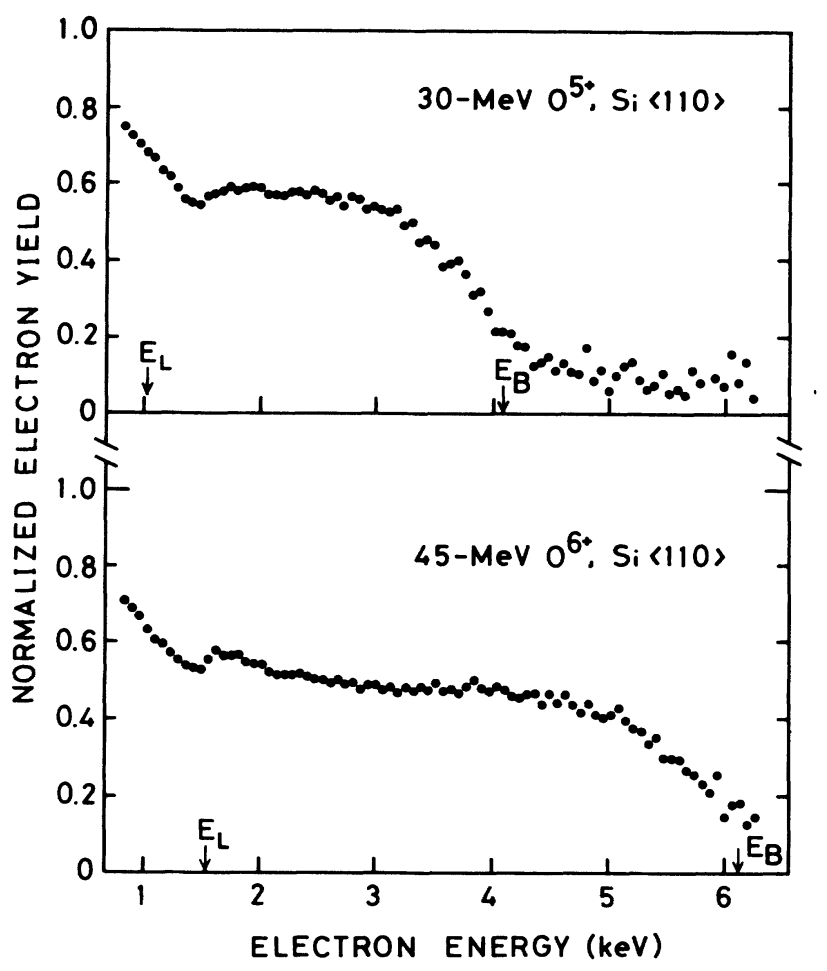

FIG. 3. Normalized electron yields (i.e., the ratio of channeling to random yield) for $30-\mathrm{MeV} \mathrm{O}^{5+}$ and $45-\mathrm{MeV} \mathrm{O}^{6+}$ under $\mathrm{Si}$ $\langle 110\rangle$ channeling conditions. $E_{L}$ and $E_{B}$ indicate the loss-peak energy and the binary-peak energy, respectively (see text).

yields (normalized to the random cases), i.e., the ratio of the channeling to random yield for $30-\mathrm{MeV} \mathrm{O}^{5+}$ and 45$\mathrm{MeV} \mathrm{O}^{6+}$ under $\mathrm{Si}\langle 110\rangle$ channeling conditions. $E_{L}$ and $E_{B}$ shown in Fig. 3 indicate the loss-peak energy, i.e., the kinetic energy of a free electron running at the ion velocity, and the binary-peak energy, i.e., the maximum energy transferred to a free electron at rest, respectively. We see from Fig. 3 that marked plateaus, which correspond to a constant decrease in the electron yield, ${ }^{10}$ appear in between $E_{L}$ and $E_{B}$, as will be discussed in Sec. IV A. The plateau region increases with increasing the ion energy since $E_{L}$ and $E_{B}$ are proportional to the ion energy.

Such a constant decrease was also observed for other ions and targets. Figures 4-6 show the results for 3.75$\mathrm{MeV} / \mathrm{amu} \mathrm{He}^{2+}, \mathrm{C}^{6+}\left(\right.$ also $\mathrm{C}^{4+}$ ), and $\mathrm{O}^{8+}$, and for 6.25and 7.5-MeV/amu $\mathrm{He}^{2+}$ incident on $\mathrm{Si}$ and $\mathrm{GaAs}$ targets. For $3.75-\mathrm{MeV} / \mathrm{amu}$ ions, the constant decrease is expected to occur in between $E_{L}=2.0 \mathrm{keV}$ and $E_{B}=8.1$ keV. Actually, each pair of spectra (measured up to about $6 \mathrm{keV}$ ) for the channeling and random case, plotted in logarithmic scale, overlaps at high-electron energies when shifted vertically, indicating the constant decrease. The charge state of the carbon and oxygen ions only affects the low-energy yield in the spectra. This is typically seen in Fig. 6, where the electron yields for $\mathrm{C}^{4+}$ and $\mathrm{C}^{6+}$ are obtained for the same number of incident carbon ions. The enhancement of the electron yield for $\mathrm{C}^{4+}$ below about $3 \mathrm{keV}$ results from the electron loss of $\mathrm{C}^{4+}$.

The constant decrease in the electron yield depends on the channeling direction. Figure 7 shows such depen- 


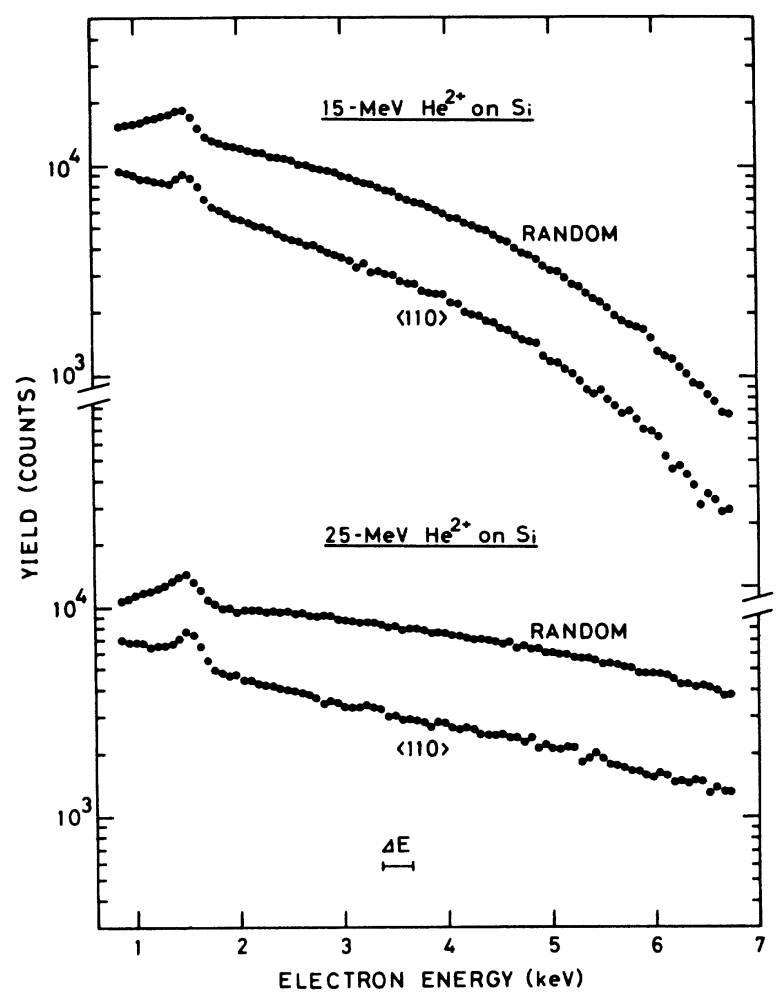

FIG. 4. Energy spectra of secondary electrons for $\mathrm{Si}\langle 110\rangle$ channeling and random incidences of $15-$ and $25-\mathrm{MeV} \mathrm{He}^{2+}$. The peaks near $1.6 \mathrm{keV}$ are due to $\mathrm{Si} K$-shell Auger electrons.

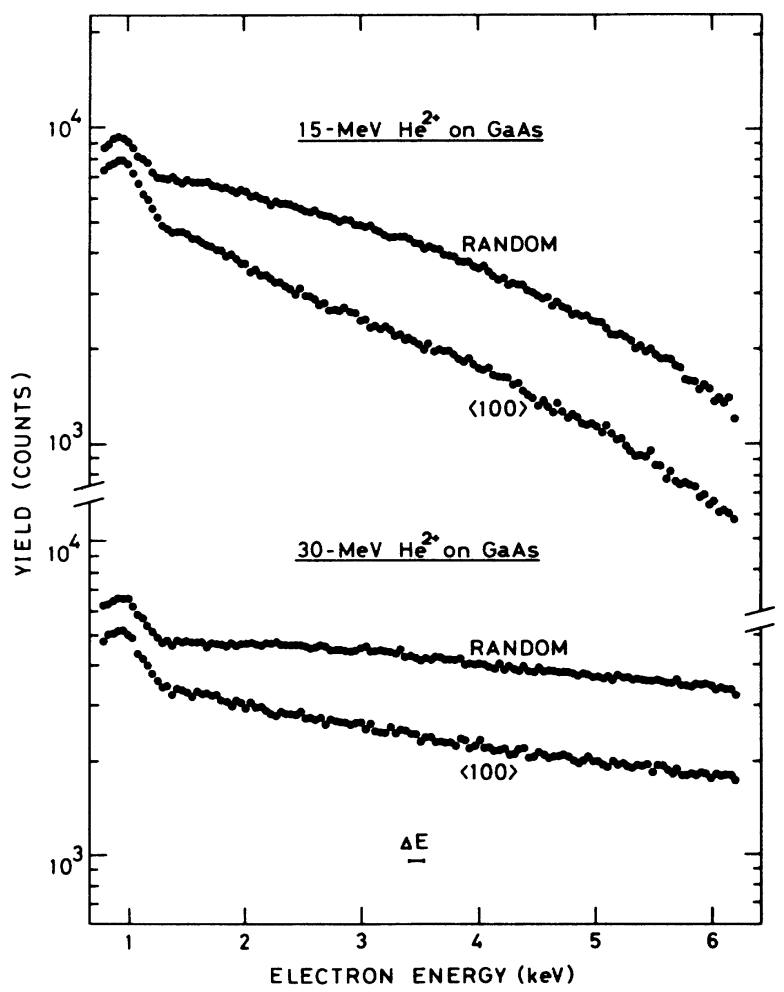

FIG. 5. Energy spectra of secondary electrons for GaAs $\langle 100\rangle$ channeling and random incidences of $15-$ and $30-\mathrm{MeV}$ $\mathrm{He}^{2+}$. The peaks near $1 \mathrm{keV}$ are due to $L$-shell Auger electrons from $\mathrm{Ga}$ and $\mathrm{As}$ atoms.

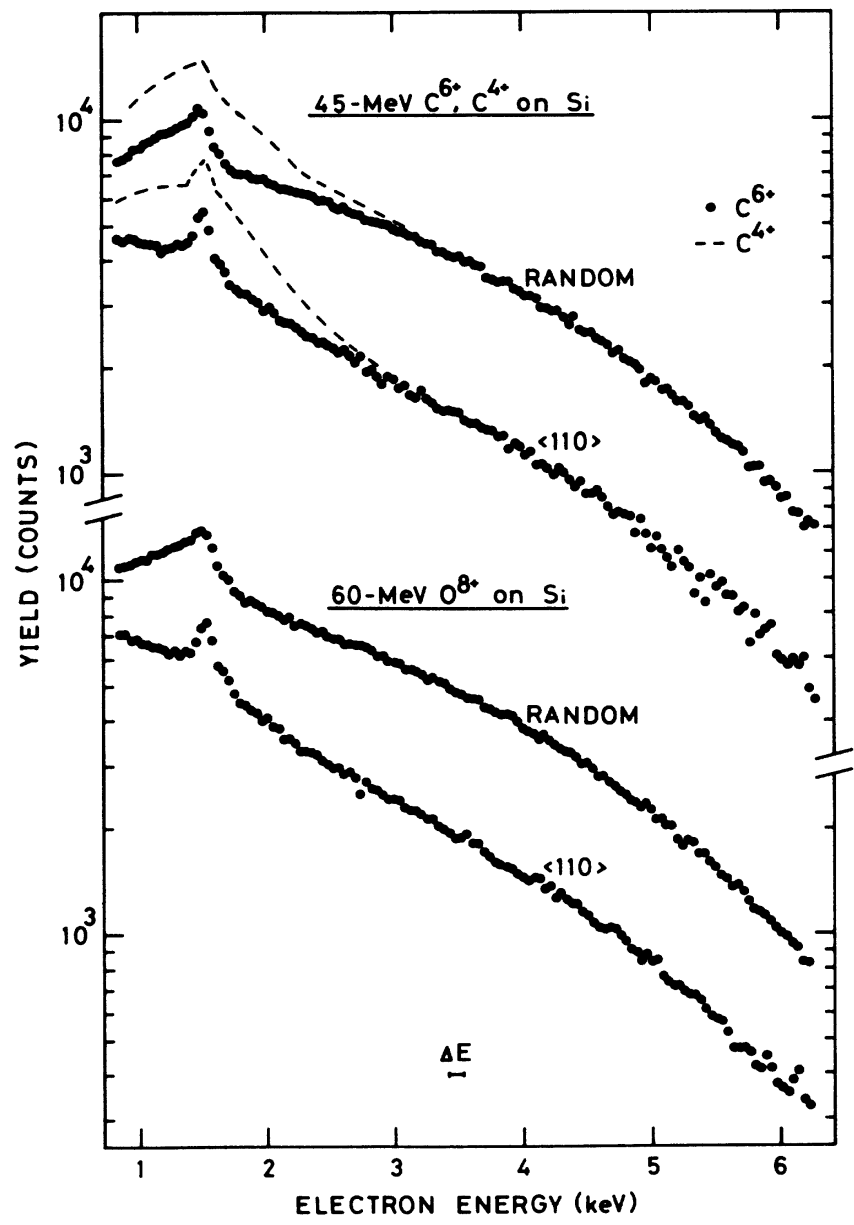

FIG. 6. Energy spectra of secondary electrons induced by C and $O$ ions of $3.75 \mathrm{MeV} / \mathrm{amu}$, measured for $\langle 110\rangle$ channeling and random incidences on Si crystals. The peaks near $1.6 \mathrm{keV}$ are due to $\mathrm{Si} K$-shell Auger electrons.

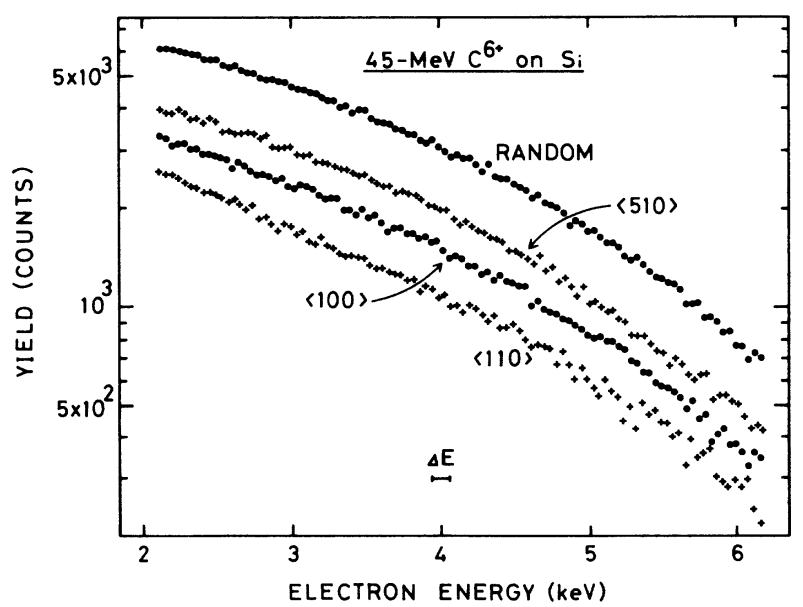

FIG. 7. Dependence of the electron-energy spectra on various axial incidences of $45-\mathrm{MeV} \mathrm{C}^{6+}$ on $\mathrm{Si}$ crystals. 
dence on the axial direction in $\mathrm{Si}$ for $\mathrm{C}^{6+}$ ions. This effect has also been observed for planar incidences of the ions.

Table I shows the constant reduction factors, which were obtained for various channeling incidences of 3.75$\mathrm{MeV} / \mathrm{amu}$ ions. For $\mathrm{Si}$, the constant reduction factors were obtained from the normalized electron yields above $3.5 \mathrm{keV}$. For $\mathrm{GaAs}$, the normalized yields have slopes of about $6 \%$ between 4 and $6 \mathrm{keV}$, so that the reduction factors were taken to be the average values at $5 \mathrm{keV}$. The uncertainty, about $\pm 5 \%$, in the values given in Table I mainly arises from the instability of the beam transport and from uncertainty in the random yield.

\section{DISCUSSION}

The ions incident in an axial direction of a crystal are deflected by successive collisions with the aligned atoms. As a result, a cone-shaped region surrounding the atomic row is formed, where the ions do not enter, so that the target electrons within this region are hardly ionized. Such shadowing effect causes the localization of ioninduced ionization of the target electrons near the surface, similar to the case of ion backscattering. ${ }^{11,12}$ Here, we analyze the results for the $3.75-\mathrm{MeV} / \mathrm{amu}$ ions in the Si axial case summarized in Table I. The planar case should be treated similarly in terms of planar shadowing.

\section{A. Production of secondary electrons}

The observed secondary electrons with energies higher than that of the Si $K$-shell Auger electrons $(1.6 \mathrm{keV})$ result from the scattering cascades of target electrons, which are triggered by the events of primary (ioninduced) scattering of electrons in the target. Therefore, the observed energy spectra of secondary electrons depend on the energy distribution of the primarily scattered electrons. Both inner-shell and valence electrons, when scattered in the target, contribute to this energy distribution in the energy range below about $E_{B}$. However, for energies above about $E_{B}$ there is a dominant contribution from inner-shell electrons that have high binding energies, ${ }^{13}$ e.g., $K$-shell electrons for $\mathrm{Si}$ target. This effect is clearly seen in Fig. 3, in which the yield ratios abruptly

TABLE I. The values of $W$, the constant reduction factor in the electron yields under channeling conditions, obtained from the measurements for various $3.75-\mathrm{MeV} / \mathrm{amu}$ ions incident on $\mathrm{Si}$ and $\mathrm{GaAs}$ targets (for GaAs, see text). The uncertainty is about $\pm 5 \%$. For the $\mathrm{Si}\langle 111\rangle$ axis, $d$ is taken to be the average between the two kinds of interatomic distances.

\begin{tabular}{lccc}
\hline \hline Channel & $\mathrm{He}^{2+}$ & $\mathrm{C}^{6+}$ & $\mathrm{O}^{8+}$ \\
\hline $\mathrm{Si}\langle 110\rangle(d=3.84 \AA)$ & 0.38 & 0.36 & 0.38 \\
$\mathrm{Si}\langle 111\rangle(d=4.70 \AA)$ & 0.47 & & \\
$\mathrm{Si}\langle 100\rangle(d=5.43 \AA)$ & 0.51 & 0.48 & 0.49 \\
$\mathrm{Si}\langle 310\rangle(d=8.59 \AA)$ & 0.61 & 0.60 & 0.57 \\
$\mathrm{Si}\langle 510\rangle(d=13.84 \AA)$ & 0.68 & 0.63 & 0.68 \\
$\mathrm{Si}(110)$ plane & 0.65 & 0.66 & \\
$\mathrm{Si}(100)$ plane & 0.76 & 0.75 & \\
$\mathrm{GaAs}\langle 100\rangle(d=5.65 \AA)$ & 0.46 & & 0.47 \\
\hline \hline
\end{tabular}

decrease near $E_{B}$ with increasing electron energy. It is evident that this trend of the electron yield above $E_{B}$ stems from the strong shadowing effect on the $K$-shell electrons in Si. On the other hand, the plateau regions in Fig. 3 should mainly come from the $L$-shell electrons in Si since the shadowing effect is weaker for the outer $L$ shell than for the inner $K$ shell.

For electron energies near or below $E_{L}$, a different mechanism contributes to the production of secondary electrons. This contribution which is due to the loss electrons released from the bound states of the projectile ions roughly gives the lower end of the plateau region of the normalized yield.

It should be noted that Auger electrons do not contribute to the energy region of interest because the energy of $\mathrm{Si} K$-shell (highest-energy) Auger electron is only 1.6 $\mathrm{keV}$; this results in the smooth energy spectra observed.

\section{B. Analysis of the yield ratios}

We derive an expression for the constant reduction factor in the electron yields $W$ with a special interest in the dependence of $W$ on various parameters.

The shadowing effect is described as the successive increase in impact parameter in a sequence of soft elastic collisions between the incident ion and aligned atoms, although this increase is somewhat disturbed by the thermal displacement of atoms, which is effectively static for $\mathrm{MeV}$ fast ions. ${ }^{5}$ In the collision sequence, each soft collision of impact parameter $b$ causes an increase in the impact parameter for the next soft collision by $\phi(b) d$, where $\phi(b)$ is the scattering angle in the laboratory frame and $d$ is the interatomic distance along the row. For a screened potential (e.g., Thomas-Fermi potential) with a screening distance $a, \phi(b) d$ is given by ${ }^{14}$

$\phi(b) d=Z_{1} Z_{2} e^{2} d g(b / a) / E_{0} b=R^{2} g(b / a) / 4 b$,

where $Z_{1}$ and $Z_{2}$ are the atomic numbers of the projectile and target, respectively, $e$ is the electronic charge, $E_{0}$ is the ion energy, and the parameter $g(b / a)$, which is less than unity, represents the correction of the scattering angle to the case of the pure Coulomb potential $(g=1) .^{15}$

$$
R\left(=\left[4 Z_{1} Z_{2} e^{2} d / E_{0}\right]^{1 / 2}\right)
$$

represents the shadow cone radius at the distance $d$ when a parallel beam is incident on an isolated atom. ${ }^{12}$ The screening distance is taken to be $\mathrm{be}^{5} a=0.8853 a_{0} Z_{2}^{-1 / 3}$, with $a_{0}$ being the Bohr radius, since in the present case the ions are highly ionized in the target. ${ }^{16}$ Equation (1) indicates that for a given target crystal the successive impact-parameter increase, i.e., the shadowing effect is determined by the parameter $R$.

Next, we derive an expression for the ratio $W$. Because the number of target atoms that are effective for producing primarily scattered electrons is restricted through the shadowing effect, the effective number of target atoms $N$ (atoms/row) is a function of $R$. Therefore, the effective target thickness for the shadowing case is expressed as $N(R) d$. We assume that the observed yield of secondary electrons in the shadowing case is proportional to the effective target thickness. This linearity enables a deriva- 
tion of $W$ without knowing the details of the production mechanism of observed secondary electrons; we may simply write

$$
W=N(R) d / t, \text { i.e., } W / d=N(R) / t
$$

where the parameter $t$ has been introduced as the effective target thickness for random incidences. The thickness $t$ corresponds to the depth within which the electron yield is proportional to the number of target atoms, like in the case of gas targets.

The value of $W$ given by Eq. (2) is independent of the observation angle of secondary electrons. Therefore, the present discussion of $\boldsymbol{W}$ is also applicable to observations at various angles, as in earlier measurements. ${ }^{6,7}$

\section{Scaling law of $\boldsymbol{W}$}

For fast ions (e.g., MeV/amu energy range), the ioninduced transition probability of a target electron from the initial to the final (scattering) state is expressed as $Z_{1}^{2} f(v)$ with $f(v)$ being the corresponding transition probability for protons with the same velocity, according to the semiclassical approximation model. ${ }^{17,18}$ This means that the parameter $Z_{1}$ constitutes only a scaling factor for the number of scattered electrons, and that the parameter $t$ in Eq. (2) is determined only by the ion's velocity $v$ irrespective of ion species. Therefore, it is concluded from Eq. (2) that the values of $W / d$ obtained for various ions of equal velocity can be universally scaled as a function of the parameter $R$. It should be noted that this scaling law essentially corresponds to a special case of the universal plot suggested by Feldman, for the surface-peak intensity observed in ion backscattering spectra. ${ }^{11,12}$

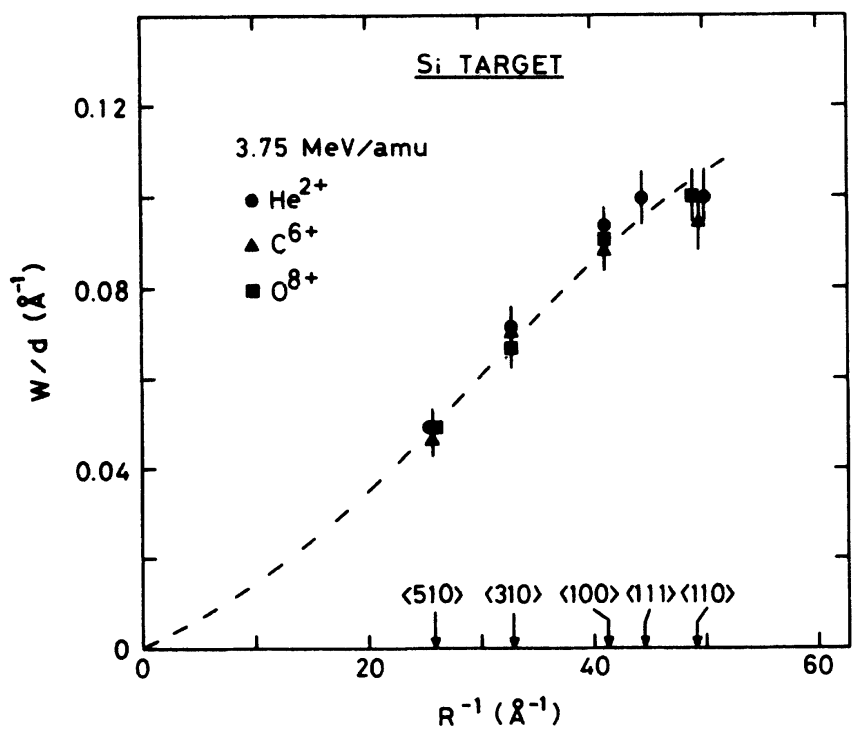

FIG. 8. Plot of $W / d$ against $R^{-1}$. The dashed curve, which is drawn to guide the eye, represents the universal curve. Note that the curve should approach the origin of the figure, corresponding to the limit of high-index axis, i.e., $d=\infty$.

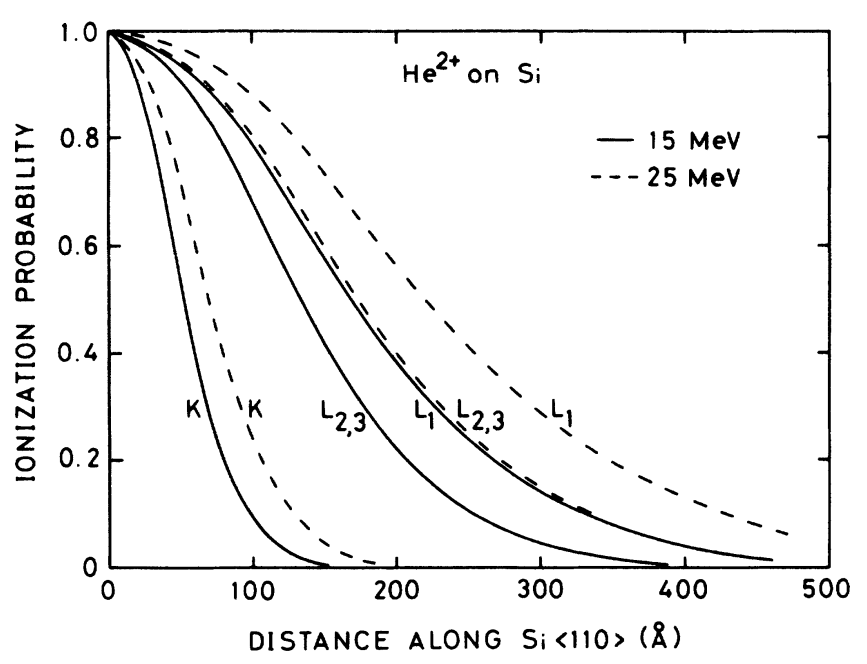

FIG. 9. Calculated ionization probabilities for various shells of $\mathrm{Si}$ for $\langle 110\rangle$ incidence of $15-$ and $25-\mathrm{MeV} \mathrm{He}^{2+}$, which are normalized to those of the surface atoms, respectively.

We use $R^{-1}$, rather than $R$, as the scaling parameter only for convenience. Figure 8 shows the plot of $W / d$ against $R^{-1}$, where the universal curve is shown by the dashed curve. This curve demonstrates that the effective number of target atoms increases with decreasing shadow cone radius.

\section{Estimate of the parameter $t$}

The analysis presented here includes an unknown parameter $t$, which provides a measure of the target thickness sensitive to the shadowing effect. Thus, it should be of fundamental importance to estimate $t$ for the present case.

Figure 9 shows the calculated ionization probabilities for $K, L_{1}$, and $L_{2,3}$ shells of $\mathrm{Si}$ for the $\langle 110\rangle$ incidence of 15- and 25-MeV $\mathrm{He}^{2+}$, normalized to those of the surface atom, respectively. The impact-parameter dependence of the ionization probability was taken from the numerical tables of Hansteen et al. ${ }^{18}$ The details of the calculation, which is based on classical ion trajectories, have been published previously. ${ }^{7-9}$ It should be noted that the results shown in Fig. 9 hold for any ion with the same value of $Z_{1} / E_{0}$ (and therefore, $R$ ), if it is fully ionized in the target and if the perturbation treatment ${ }^{18}$ of the ioninduced ionization is valid. We may assume that the observed electron yield mainly results from the ionization of the $L$ shells, although neglecting the contribution of the few valence electrons should lead to an underestimate of the effective target thickness, and therefore, of $t$. Since for $15-\mathrm{MeV} \mathrm{He}^{2+}$ the ionization probability for the easily-ionized $L_{2,3}$ shell decreases to $50 \%$ at about $130 \AA$, as seen in Fig. 9, we obtain $t \simeq 340 \AA$ for $15-\mathrm{MeV} \mathrm{He}^{2+}$ (i.e., also for 3.75-MeV/amu $\mathrm{C}$ and $\mathrm{O}$ ions) from Eq. (2) by using $N(R) d \simeq 130 \AA$ and the observed value of $W=0.38$. Similarly, we can estimate that $t \simeq 470 \AA$ for $25-\mathrm{MeV} \mathrm{He}^{2+}$ from the values of $N(R) d \simeq 170 \AA$ and $W=0.36$. 


\section{CONCLUDING REMARKS}

The yield of secondary electrons emitted under channeling conditions is a sensitive probe for the crystal quality near the surfaces. The value of $W$, the well-defined yield ratio between the channeling and random cases, provides a measure for the quality. An important application would lie in the characterization of thin epitaxially-grown crystals.

To obtain a better understanding of the shadowing effect on the production of secondary electrons, further investigation is necessary on secondary electrons with energies above $E_{B}$, which suffer a strong shadowing effect.

\section{ACKNOWLEDGMENTS}

We thank T. Uchino, O. Eryu, T. Gochou, and $\mathrm{K}$. Takahashi for their assistance in the measurements. We are also grateful to J. Lindhard, J. P. Biersack, and $Y$. Yamazaki for valuable comments on this work and to $\mathbf{W}$. Galster for a critical reading of the manuscript. This work was supported in part by a Grant-in-Aid for Special-Project Research on ion beam interactions with solids from the Ministry of Education, Science, and Culture.
${ }^{1}$ G. D. Magnuson and C. E. Carlston, Phys. Rev. 129, 2409 (1963).

${ }^{2}$ N. Colombie, B. Fagot, and C. Fert, Radiat. Eff. 2, 31 (1969).

${ }^{3}$ B. A. Brushilovsky and V. A. Molchanov, Radiat. Eff. 23, 131 (1974).

${ }^{4}$ N. Benazeth, Nucl. Instrum. Methods 194, 405 (1982).

${ }^{5}$ D. S. Gemmell, Rev. Mod. Phys. 46, 129 (1974).

6J. R. MacDonald, L. C. Feldman, P. J. Silverman, J. A. Davies, K. Griffiths, T. E. Jackman, P. R. Norton, and W. N. Unertl, Nucl. Instrum. Methods 218, 765 (1983).

${ }^{7}$ H. Kudo, D. Schneider, E. P. Kanter, P. W. Arcuni, and E. A. Johnson, Phys. Rev. B 30, 4899 (1984).

${ }^{8}$ H. Kudo, K. Murakami, K. Takita, K. Masuda, S. Seki, K. Shima, H. Itoh, and T. Ipposhi, Jpn. J. Appl. Phys. 24, 1440 (1985).

${ }^{9}$ H. Kudo, K. Shima, K. Takita, K. Masuda, K. Murakami, H. Itoh, T. Ipposhi, and S. Seki, Jpn. J. Appl. Phys. 25, 1751 (1986).

${ }^{10}$ The observed electron yield is proportional to $E \eta(E)$, where $E$ is the electron energy, and $\eta(E)$ is the efficiency of the electron multiplier (the factor $E$ arises from the spectrometer's acceptance). Note that this $E$ dependence is canceled out in the yield ratios obtained.

${ }^{11}$ L. C. Feldman, Nucl. Instrum. Methods 191, 211 (1981).

${ }^{12}$ L. C. Feldman, J. W. Mayer, and S. T. Picraux, Material Analysis by Ion Channeling (Academic, New York, 1982), Chap. 1.

${ }^{13} \mathrm{See}$, for example, binary-encounter calculations by F. Folkmann, C. Gaarde, T. Huus, and K. Kemp, Nucl. Instrum. Methods 116, 487 (1974).

${ }^{14}$ For example, C. Lehmann, Interaction of Radiation with Solids and Elementary Defect Production (North-Holland, New York, 1977), Part 1. Note that small-angle scattering is independent of the target mass.

15J. Lindhard, V. Nielsen, and M. Scharff, K. Dan Vidensk. Selsk. Mat. Fys. Medd. 36, No. 10 (1968), Sec. 3.

${ }^{16}$ K. Shima, T. Mikumo, and H. Tawara, At. Data Nucl. Data Tables 34, 357 (1986), and references therein.

${ }^{17}$ J. Bang and J. M. Hansteen, K. Dan Vidensk. Selsk. Mat. Fys. Medd. 31, No. 13 (1959).

18J. M. Hansteen, O. M. Johnsen, and L. Kocbach, At. Data Nucl. Data Tables 15, 305 (1975). 